\title{
Higgs-portal dark matter in the nonlinear MSSM
}

\author{
Masato Arai ${ }^{1, *}$ and Nobuchika Okada $\oplus^{2, \dagger}$ \\ ${ }^{1}$ Faculty of Science, Yamagata University, Yamagata 990-8560, Japan \\ ${ }^{2}$ Department of Physics and Astronomy, University of Alabama, Tuscaloosa, Aalabama 35487, USA
}

(Received 17 August 2020; accepted 8 January 2021; published 25 January 2021)

\begin{abstract}
Supersymmetric (SUSY) extension of the Standard Model (SM) is a primary candidate for new physics beyond the SM. If SUSY breaking scale is very low, for example, the multi-TeV range, and the SUSY breaking sector, except for the Goldstino (gravitino), is decoupled from the low energy spectrum, the hidden sector effect in the minimal SUSY SM (MSSM) is well described by employing the Goldstino chiral superfield $(X)$ with the nilpotent condition of $X^{2}=0$. Although this so-called "nonlinear MSSM" (NL-MSSM) provides a variety of interesting phenomenologies, there is a cosmological problem that the lightest superpartner gravitino is too light to be the major component of the dark matter (DM) in our Universe. To solve this problem, we propose a minimal extension of the NL-MSSM by introducing a parity-odd SM singlet chiral superfield $(\Phi)$. We show that the interaction of the scalar component in $\Phi$ with the MSSM Higgs doublets is induced after eliminating $F$ component of the Goldstino superfield, and the lightest real scalar in $\Phi$ plays the role of the Higgs-portal DM. With a suitable choice of the model parameters, a successful Higgs-portal DM scenario can be realized. In addition, if SUSY breaking scale lies in the multi-TeV range, the SM-like Higgs boson mass of $125 \mathrm{GeV}$ can be achieved by the tree-level Higgs potential through the low-scale SUSY breaking effect.
\end{abstract}

DOI: 10.1103/PhysRevD.103.015027

\section{INTRODUCTION}

Although the current experimental data show no plausible evidence of new physics beyond the Standard Model (SM), the minimal supersymmetric (SUSY) extension of the SM (MSSM) is still a primary candidate for new physics. As has been well-known and intensively studied, the MSSM not only provides us with a solution to the gauge hierarchy problem but also offers a variety of interesting phenomenologies, such as the origin of the electroweak symmetry breaking from SUSY breaking, the SM-like Higgs boson mass prediction with soft SUSY breaking parameters, the lightest superpartner (LSP) as a natural candidate for the dark matter (DM) in our Universe, and the grand unified theory paradigm with the successful unification of the three SM gauge couplings at the scale of $\mathcal{O}\left(10^{16} \mathrm{GeV}\right)$. Many ongoing and planned experiments will continue searching for the MSSM, or in more general, supersymmetric theories beyond the SM.

\footnotetext{
arai@sci.kj.yamagata-u.ac.jp

†okadan@ua.edu
}

Published by the American Physical Society under the terms of the Creative Commons Attribution 4.0 International license. Further distribution of this work must maintain attribution to the author(s) and the published article's title, journal citation, and DOI. Funded by SCOAP ${ }^{3}$.
In phenomenologically viable models, SUSY is spontaneously broken in the hidden sector, and the SUSY breaking effects are mediated to the MSSM sector by a certain mechanism for generating soft SUSY breaking terms in the MSSM. Associated with spontaneous SUSY breaking, a massless fermion called Goldstino emerges due to the Nambu-Goldstone theorem, and it is absorbed into the spin-1/2 component of the spin-3/2 massive gravitino in supergravity. The gravitino mass is characterized by the SUSY breaking order parameter $f$ and the reduced Planck mass of $M_{P}=2.43 \times 10^{19} \mathrm{GeV}$ as $m_{3 / 2} \simeq f / M_{P}$. It is possible that SUSY breaking occurs at a very low energy (see, for example, Ref. [1]). If this is the case, the gravitino becomes the LSP and is involved in phenomenology at low energies. For example, if the SUSY breaking scale lies in the multi-TeV range, the LSP gravitino is extremely light with its mass of $\mathcal{O}(\mathrm{meV})$. Assuming the decoupling of the hidden sector fields except for the light gravitino (or, equivalently, Goldstino), the low energy effective theory involving the very light gravitino can be described by employing a Goldstino chiral superfield $X$ with the nilpotent condition $X^{2}=0$ [2-4]. With this formalism, the phenomenology of the MSSM with the Goldstino superfield has been studied in detail [5-7] (see also Ref. [8] for the phenomenology in a more general setup). This framework is the so-called nonlinear MSSM (NL-MSSM). Interestingly, it has been shown that if the SUSY breaking 
scale lies in the multi-TeV range, the SM-like Higgs boson receives a sizable contribution to its mass at the tree-level after eliminating $F$ component of the Goldstino superfield, and as a result, the Higgs boson mass of around $125 \mathrm{GeV}$ can be achieved by the tree-level Higgs potential. This is in sharp contrast with the usual MSSM in which the $125 \mathrm{GeV}$ SM-like Higgs boson mass is reproduced by quantum corrections through scalar top quarks with the mass larger than multi-TeV. In the viewpoint of the collider physics, the NL-MSSM has an advantage that the scalar top quarks can be sufficiently light to be explored in the near future.

The SUSY breaking order parameter $\sqrt{f} \lesssim \mathcal{O}(100) \mathrm{TeV}$ gives the extremely light gravitino with mass $m_{3 / 2} \lesssim 10 \mathrm{eV}$ in the NL-MSSM. Although such a light gravitino is harmless in the phenomenological point of view (see, for example, Ref. [9]), its relic density is far below the observed dark matter (DM) density. Even if the observed relic density is achieved by some nonstandard thermal history of the Universe, the very light gravitino is likely to be a hot DM and prevents the formation of the observed structure of the Universe. Therefore, for the completion of the NL-MSSM, we should consider an extension of the model which can supplement the model with a suitable DM candidate. In this paper, we propose a minimal extension of the NL-MSSM by introducing a $Z_{2}$-parity odd SM gauge singlet chiral superfield $\Phi$ and show that the lightest scalar component in $\Phi$ plays the role of the Higgs-portal DM $[10,11]^{1}$ through its coupling with the MSSM Higgs doublets induced by the Goldstino superfield. With a suitable choice of the model parameters, we can realize a phenomenologically viable Higgs-portal DM scenario. If SUSY breaking scale lies in the multi-TeV range, the SM-like Higgs boson mass of $125 \mathrm{GeV}$ can be achieved by the tree-level Higgs potential through the low-scale SUSY breaking effect.

\section{NL-MSSM AND THE HIGGS BOSON MASS}

We first present the basic formalism of the NL-MSSM and show how the $125 \mathrm{GeV}$ SM-like Higgs boson mass can be achieved in the framework. We begin with the Goldstino effective Lagrangian of the form [4],

$$
\mathcal{L}_{X}=\int d^{4} \theta X^{\dagger} X+\left(\int d^{2} \theta f X+\text { H.c. }\right),
$$

where $X$ is a Goldstino chiral superfield, and $f$ is the SUSY breaking order parameter in the hidden sector. Although the stability of the hidden sector scalar potential needs an extension of the above minimal Kähler potential, this Lagrangian is enough to understand the essence of the formalism. The Goldstino chiral superfield is subject to the nilpotent condition [2-4],

\footnotetext{
${ }^{1}$ For a recent review, see Ref. [12] and references therein.
}

$$
X^{2}=0
$$

which leads us to the expression of the superfield with the components,

$$
X=\frac{\psi_{X} \psi_{X}}{2 F_{X}}+\sqrt{2} \theta \psi_{X}+\theta \theta F_{X}
$$

The scalar component in the Goldstino superfield is to be integrated out in the low energy effective theory, and under the nilpotent condition, it is replaced by the bilinear term of the Goldstino fields. In fact, substituting Eq. (3) into Eq. (1) and eliminating the auxiliary field $F_{X}$, we recover the Volkov-Akulov Lagrangian [13].

In the superfield formalism, the spurion technique is a simple way to introduce the soft SUSY breaking terms to the MSSM Lagrangian. We introduce a dimensionless and SM-singlet spurion field of the form, $Y=\theta^{2} m_{\text {soft }}$, where $m_{\text {soft }}$ is a generic notation for the soft terms (denoted $m_{1,2,3}, m_{\Psi}, m_{\lambda_{a}}$ in the following) and attach it to any SUSY operators in the MSSM. The recipe to obtain the NL-MSSM is to replace the spurion by the Goldstino superfield as [4]

$$
Y \rightarrow \frac{m_{\mathrm{soft}}}{f} X
$$

We apply this rule and write the NL-MSSM Lagrangian as follows [5]:

$$
\mathcal{L}=\mathcal{L}_{0}+\mathcal{L}_{X}+\mathcal{L}_{H}+\mathcal{L}_{m}+\mathcal{L}_{A B}+\mathcal{L}_{g}
$$

In the right-hand side, the first term $\mathcal{L}_{0}$ denotes the supersymmetric part of the MSSM Lagrangian given by ${ }^{2}$

$$
\begin{aligned}
\mathcal{L}_{0}= & \sum_{\Psi, H_{u}, H_{d}} \int d^{4} \theta \Psi^{\dagger} e^{V} \Psi \\
& +\left\{\int d ^ { 2 } \theta \left[\mu_{H} H_{d} H_{u}+\lambda_{u} H_{u} Q U^{c}+\lambda_{d} Q D^{c} H_{d}\right.\right. \\
& \left.\left.+\lambda_{e} L E^{c} H_{d}\right]+ \text { H.c. }\right\} \\
& +\sum_{\mathrm{a}=1}^{3} \frac{1}{4 g_{a}^{2} \kappa} \int d^{2} \theta \operatorname{Tr}\left[W_{a}^{\alpha} W_{a \alpha}\right]+\text { H.c. }
\end{aligned}
$$

where $\Psi=Q, U^{c}, D^{c}, L, E^{c}$, the index $a=1,2,3$ denotes the SM gauge groups $S U(3), S U(2)$, and $U(1), g_{a}$ is the corresponding gauge couplings, and $\kappa=1$ for $U(1)$ and $1 / 2$ for $S U(3)$ and $S U(2)$. The vector superfield $V$ in the Kähler potential for the chiral superfields implies, for example, $V=2 V_{3}+2 V_{2}+\frac{1}{3} V_{1}$ for $Q$ etc., where

\footnotetext{
${ }^{2}$ For a concise review of the MSSM and the standard notation, see, for example, Ref. [14].
} 
$V_{a}(a=1,2,3)$ denote the vector superfields of the corresponding SM gauge groups. $\mathcal{L}_{X}$ is the hidden sector Lagrangian already introduced in Eq. (1). $\mathcal{L}_{H}$ is the Higgs sector Lagrangian involving the Goldstino sueprfield,

$$
\begin{aligned}
\mathcal{L}_{H}= & -\frac{m_{1}^{2}}{f^{2}} \int d^{4} \theta\left(X^{\dagger} X\right) H_{d}^{\dagger} e^{V} H_{d} \\
& -\frac{m_{2}^{2}}{f^{2}} \int d^{4} \theta\left(X^{\dagger} X\right) H_{u}^{\dagger} e^{V} H_{u} .
\end{aligned}
$$

The matter field Lagrangian involving the Goldstino superfield is given by

$$
\mathcal{L}_{m}=-\sum_{\Psi}\left(\frac{m_{\Psi}^{2}}{f^{2}}\right) \int d^{4} \theta\left(X^{\dagger} X\right) \Psi^{\dagger} e^{V} \Psi
$$

The bilinear and trilinear SUSY breaking couplings are given by $\mathcal{L}_{A B}$,

$$
\begin{aligned}
\mathcal{L}_{A B}= & \frac{m_{3}^{2}}{f} \int d^{2} \theta X H_{d} H_{u}+\text { H.c. } \\
& +\int d^{2} \theta X\left\{\lambda_{u}\left(\frac{A_{u}}{f}\right) U^{c} H_{u} Q+\lambda_{d}\left(\frac{A_{d}}{f}\right) D^{c} H_{d} Q\right. \\
& \left.+\lambda_{e}\left(\frac{A_{e}}{f}\right) E^{c} H_{d} L\right\}+ \text { H.c. }
\end{aligned}
$$

The last term $\mathcal{L}_{g}$ denotes the gauge sector Lagrangian given by

$$
\mathcal{L}_{g}=\sum_{a=1}^{3} \frac{1}{4 g_{a}^{2} \kappa} \frac{2 m_{\lambda_{a}}}{f} \int d^{2} \theta X \operatorname{Tr}\left[W_{a}^{\alpha} W_{a \alpha}\right]+\text { H.c. }
$$

We focus on the Higgs potential in the NL-MSSM, which is read off from $\mathcal{L}_{0}+\mathcal{L}_{X}+\mathcal{L}_{H}+\mathcal{L}_{A B}$,

$$
V=V_{\mathrm{SUSY}}+V_{\mathrm{soft}},
$$

where

$$
\begin{gathered}
V_{\text {SUSY }}=\mu_{H}^{2}\left(\left|H_{u}\right|^{2}+\left|H_{d}\right|^{2}\right)+\frac{g_{Z}^{2}}{8}\left(\left|H_{u}\right|^{2}-\left|H_{d}\right|^{2}\right)^{2} \\
+\frac{g_{2}^{2}}{2}\left|H_{u}^{\dagger} H_{d}\right|^{2} \\
V_{\text {soft }}=\frac{\left|f+\frac{m_{3}^{2}}{f} H_{u} H_{d}\right|^{2}}{1-\frac{m_{1}^{2}}{f^{2}}\left|H_{d}\right|^{2}-\frac{m_{2}^{2}}{f^{2}}\left|H_{u}\right|^{2}}
\end{gathered}
$$

with $g_{Z}^{2} \equiv g_{1}^{2}+g_{2}^{2}$. We express the up-type Higgs and down-type Higgs doublets as

$$
\begin{gathered}
H_{u}=\left(\begin{array}{c}
H^{+} \\
\frac{1}{\sqrt{2}}\left(v_{u}+R_{u}+i I_{u}\right)
\end{array}\right), \\
H_{d}=\left(\begin{array}{c}
\frac{1}{\sqrt{2}}\left(v_{d}+R_{d}+i I_{d}\right) \\
H^{-}
\end{array}\right),
\end{gathered}
$$

where $v_{u}=v \sin \beta, v_{d}=v \cos \beta$ with $v=246 \mathrm{GeV}, H^{ \pm}$ are charged Higgs fields, and $R_{u}, I_{u}, R_{d}, I_{d}$ are real scalar fields. Substituting them into the Higgs potential, we derive the stationary conditions,

$$
\begin{aligned}
\left.\frac{\partial V}{\partial R_{u}}\right|_{0}= & \frac{v}{4}\left\{4 \mu_{H}^{2} \sin \beta-2 M_{Z}^{2} \cos 2 \beta \sin \beta\right. \\
& \left.-\frac{2 \mathcal{A} m_{3}^{2} \cos \beta}{\mathcal{B}}+\frac{\mathcal{A}^{2} m_{2}^{2} \sin \beta}{\mathcal{B}^{2}}\right\}=0,
\end{aligned}
$$

$$
\begin{aligned}
\left.\frac{\partial V}{\partial R_{d}}\right|_{0}= & \frac{v}{4}\left\{4 \mu_{H}^{2} \cos \beta+M_{Z}^{2}(\cos \beta+\cos 3 \beta)\right. \\
& \left.-\frac{2 \mathcal{A} m_{3}^{2} \sin \beta}{\mathcal{B}}+\frac{\mathcal{A}^{2} m_{1}^{2} \cos \beta}{\mathcal{B}^{2}}\right\}=0,
\end{aligned}
$$

where $\left.\right|_{0}$ means that all the fields are taken to be zero, and

$$
\begin{aligned}
M_{Z}^{2} & =\frac{1}{4} g_{Z}^{2} v^{2}, \\
\mathcal{A} & =4 f^{2}+m_{3}^{2} v^{2} \sin 2 \beta, \\
\mathcal{B} & =-2 f^{2}+m_{1}^{2} v^{2} \cos ^{2} \beta+m_{2}^{2} v^{2} \sin ^{2} \beta .
\end{aligned}
$$

The other stationary conditions such as $\left.\frac{\partial V}{\partial I_{u}}\right|_{0}$ are automatically satisfied. The mass matrix of the $C P$-even Higgs bosons is given by

$$
\mathcal{M}_{C P-\mathrm{even}}=\left(\begin{array}{cc}
\left.\frac{\partial^{2} V}{\partial R_{d}^{2}}\right|_{0} & \left.\frac{\partial^{2} V}{\partial R_{d} \partial R_{u}}\right|_{0} \\
\left.\frac{\partial^{2} V}{\partial R_{u} \partial R_{d}}\right|_{0} & \left.\frac{\partial^{2} V}{\partial R_{u}^{2}}\right|_{0}
\end{array}\right),
$$

while the mass matrices for the $C P$-odd Higgs bosons and the charged Higgs bosons are

$$
\begin{aligned}
\mathcal{M}_{C P-\text { odd }} & =\left(\begin{array}{cc}
\left.\frac{\partial^{2} V}{\partial I_{d}^{2}}\right|_{0} & \left.\frac{\partial^{2} V}{\partial I_{d} \partial I_{u}}\right|_{0} \\
\left.\frac{\partial^{2} V}{\partial I_{u} \partial I_{d}}\right|_{0} & \left.\frac{\partial^{2} V}{\partial I_{u}^{2}}\right|_{0}
\end{array}\right), \\
\mathcal{M}_{\text {charged }} & =\left(\begin{array}{cc}
\left.\frac{\partial^{2} V}{\partial H^{-} \partial H^{-*}}\right|_{0} & \left.\frac{\partial^{2} V}{\partial H^{-} \partial H^{+}}\right|_{0} \\
\left.\frac{\partial^{2} V}{\partial H^{*} \partial H^{+*}}\right|_{0} & \left.\frac{\partial^{2} V}{\partial H^{+} \partial H^{+*}}\right|_{0}
\end{array}\right) .
\end{aligned}
$$

By using the above formulas, we numerically calculate the Higgs boson mass spectra. First, we choose appropriate values for $m_{1}, m_{2}, \tan \beta$, and $\sqrt{f}$ as the input parameters and solve the stationary conditions of Eqs. (16) and (17) to 


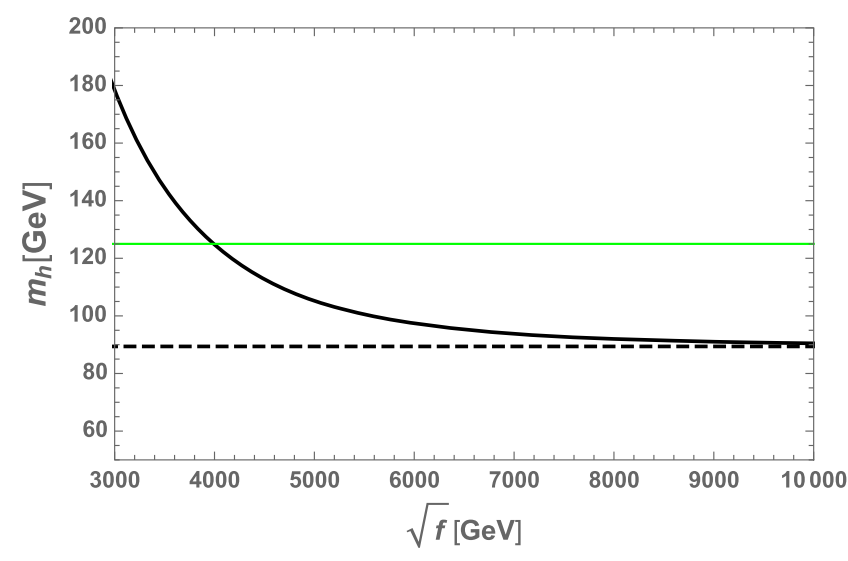

FIG. 1. The SM-like Higgs boson mass $\left(m_{h}\right)$ at the tree-level as a function of $\sqrt{f}$ (solid line), along with the standard MSSM prediction at the tree-level (dashed line), and the (green) horizontal line indicting $m_{h}=125 \mathrm{GeV}$. In this plot, we have taken $m_{1}^{2}=1000^{2} \mathrm{GeV}^{2}, m_{2}^{2}=-(2005)^{2} \mathrm{GeV}^{2}$, and $\tan \beta=10$.

fix the values of $\mu_{H}$ and $m_{3}^{2}$. We then substitute them into the Higgs potential and calculate the Higgs boson mass eigenvalues from Eqs. (19) and (20). Our results are shown in Figs. 1 and 2. The solid line in Fig. 1 shows the mass of the SM-like Higgs boson $\left(m_{h}\right)$ as a function of $\sqrt{f}$, where we have fixed $m_{1}^{2}=1000^{2} \mathrm{GeV}^{2}, m_{2}^{2}=-(2005)^{2} \mathrm{GeV}^{2}$, and $\tan \beta=10$. As $\sqrt{f}$ decreases, the SM-like Higgs boson mass increases from the standard MSSM prediction at the three level $m_{h} \simeq M_{Z} \cos 2 \beta$ (dashed line) in the limit of $\sqrt{f} \rightarrow \infty$. The (green) horizontal line indicates $m_{h}=125 \mathrm{GeV}$. We find that the main contribution for increasing the SM-like Higgs boson mass comes from the quartic coupling $\left(m_{2}^{2}\left|H_{u}\right|^{2} / f\right)^{2}$ in the series of expansion of Eq. (13), and the resultant Higgs boson mass is approximately expressed as

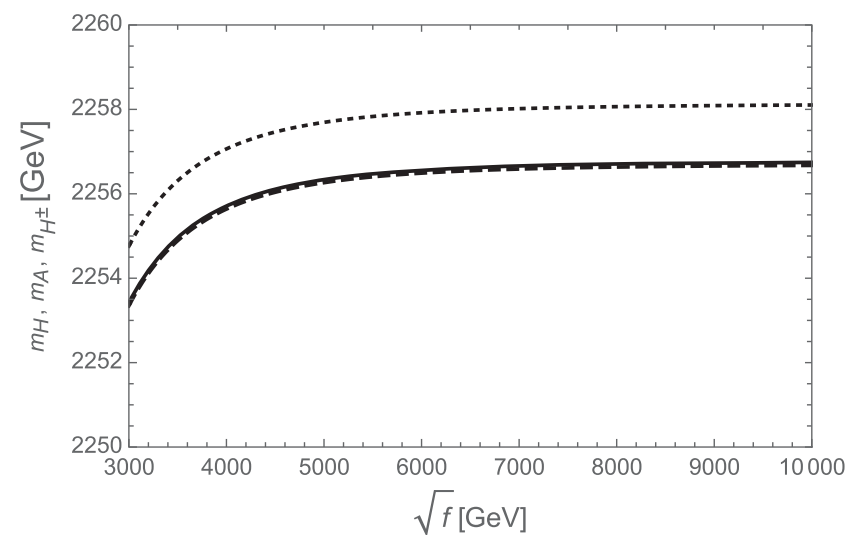

FIG. 2. Same as Fig. 1 but for the $C P$-even heavy Higgs boson mass $\left(m_{H}\right)$ (solid line), the $C P$-odd Higgs boson mass $\left(m_{A}\right)$ (dashed line), and the charged Higgs boson mass $\left(m_{H^{ \pm}}\right)$ (dotted line).

$$
m_{h}^{2} \simeq M_{Z}^{2} \cos 2 \beta+2\left(\frac{m_{2}^{2}}{f}\right)^{2} v^{2} \sin ^{2} \beta .
$$

Therefore, if the SUSY breaking scale is low enough, the SM-like Higgs boson mass of $125 \mathrm{GeV}$ is achieved by the Higgs potential at the tree level. As shown in Fig. 1, we have obtained $m_{h}=125 \mathrm{GeV}$ for $\sqrt{f}=3990 \mathrm{GeV}$. If the SUSY breaking scale is larger, the hidden sector effect on the SM-like Higgs boson mass is negligible, so that quantum corrections through heavy scalar top quarks play the crucial role to reproduce $m_{h}=125 \mathrm{GeV}$, as usual in the MSSM. Figure 2 shows the masses of the heavy neutral Higgs and the charged Higgs bosons as a function of $\sqrt{f}$ with the same inputs as in Fig. 1. The solid line depicts to the mass of the heavy $C P$-even Higgs boson $\left(m_{H}\right)$ while the dashed and dotted lines correspond to the $C P$-odd Higgs boson mass $\left(m_{A}\right)$ and the charged Higgs boson mass $\left(m_{H^{ \pm}}\right)$, respectively.

\section{MINIMAL EXTENSION WITH HIGGS-PORTAL DARK MATTER}

If the SUSY breaking scale is $\sqrt{f} \lesssim 100 \mathrm{TeV}$, the gravitino mass is found to be $m_{3 / 2} \lesssim 10 \mathrm{eV}$. Although such a light gravitino (Goldstino) is harmless in a phenomenological point of view, it is unable to be the dominant component of the DM in our Universe, and therefore, a suitable DM candidate should be supplemented to the NL-MSSM. In order to solve this problem, we propose a minimal extension of the NL-MSSM to incorporate a dark matter candidate, namely, the (scalar) Higgs-portal DM.

The Higgs-portal DM scenario is one of the simplest SM extensions to supplement the SM with a dark matter candidate. For a recent review, see Ref. [12] and references therein. In the simplest setup, we introduce an SM-singlet real scalar $(S)$ along with a $Z_{2}$ symmetry. The stability of this scalar is ensured by assigning an odd parity to it, while all the SM fields are $Z_{2}$ even. At the renormalizable level, the Lagrangian is given by

$$
\begin{aligned}
\mathcal{L}= & \mathcal{L}_{\mathrm{SM}}+\frac{1}{2}\left(\partial_{\mu} S\right)\left(\partial^{\mu} S\right)-\frac{1}{2} M_{S}^{2} S^{2}-\frac{1}{4} \lambda_{S} S^{4} \\
& -\frac{1}{4} \lambda_{H S S}\left(H^{\dagger} H\right) S^{2},
\end{aligned}
$$

where $\mathcal{L}_{\mathrm{SM}}$ is the Lagrangian of the $\mathrm{SM}$, and $H$ is the SM Higgs doublet field. After the electroweak symmetry breaking, the Lagrangian becomes

$$
\begin{aligned}
\mathcal{L}= & \mathcal{L}_{\mathrm{SM}}+\frac{1}{2}\left(\partial_{\mu} S\right)\left(\partial^{\mu} S\right)-\frac{1}{2} m_{\mathrm{DM}}^{2} S^{2}-\frac{1}{4} \lambda_{S} S^{4} \\
& -\frac{1}{4} \lambda_{H S S} v h S^{2}-\frac{1}{8} \lambda_{H S S} h^{2} S^{2},
\end{aligned}
$$

where $h$ is the physical Higgs boson, and the DM mass $m_{\mathrm{DM}}$ is given by 


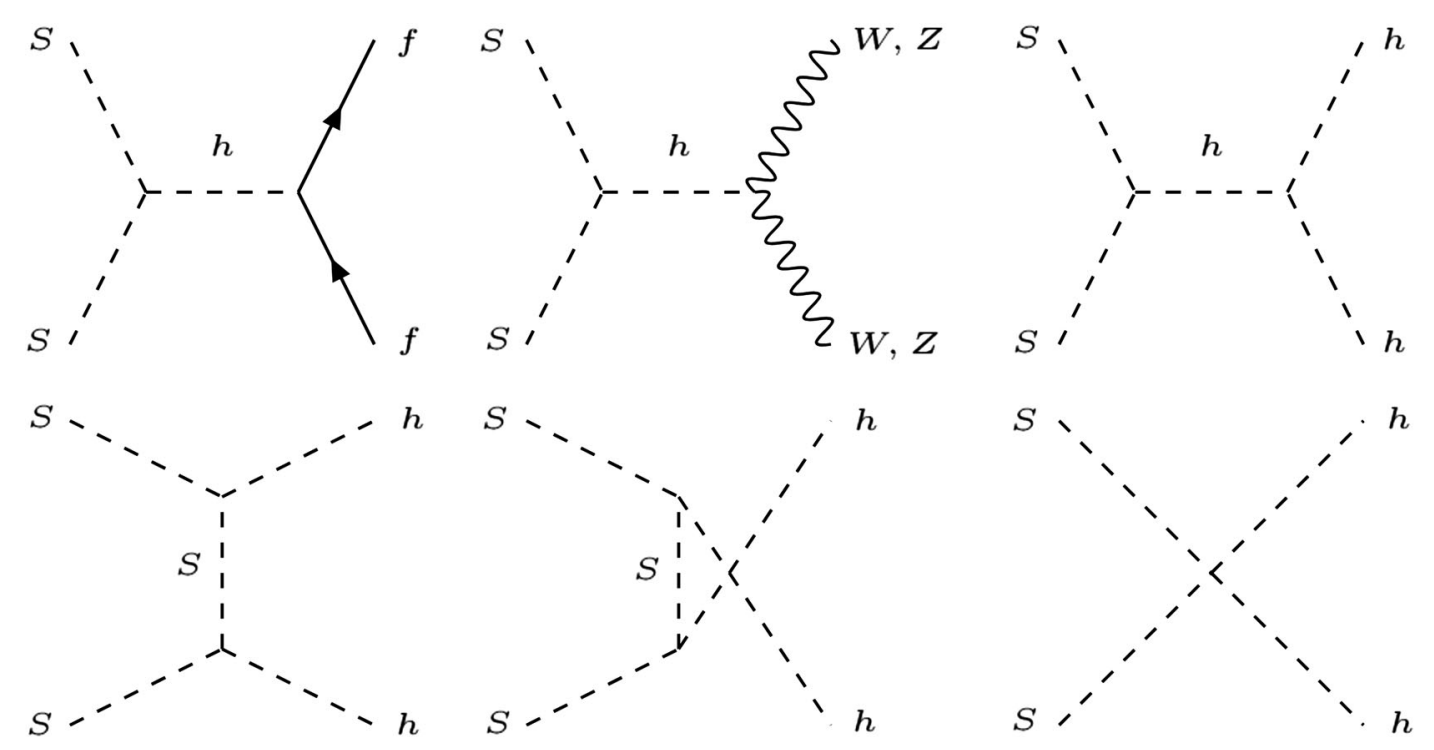

FIG. 3. Feynman diagrams for dark matter annihilations.

$$
m_{\mathrm{DM}}^{2}=M_{S}^{2}+\frac{1}{4} \lambda_{H S S} v^{2}
$$

Here, the vacuum expectation value of the Higgs field is set to be $\langle H\rangle=(0, v)^{T} / \sqrt{2}$ with $v=246 \mathrm{GeV}$. The DM phenomenology in this Higgs-portal DM scenario is controlled by only two free parameters: $m_{\mathrm{DM}}$ and $\lambda_{H S S}$.

The scalar DM $S$ annihilates into the SM particles through its coupling with the Higgs boson. The annihilation processes are shown in Fig. 3, where $W(Z)$ is the charged (neutral) weak gauge boson, and $f$ represents quarks and leptons in the SM. We evaluate the DM relic density by solving the Boltzmann equation [15],

$$
\frac{d Y}{d x}=-\frac{s\left(m_{\mathrm{DM}}\right)}{H\left(m_{\mathrm{DM}}\right)} \frac{\left\langle\sigma v_{\mathrm{rel}}\right\rangle}{x^{2}}\left(Y^{2}-Y_{E Q}^{2}\right),
$$

where the temperature of the Universe is normalized by the $\mathrm{DM}$ mass as $x=m_{\mathrm{DM}} / T, H\left(m_{\mathrm{DM}}\right)$ and $s\left(m_{\mathrm{DM}}\right)$ are the Hubble parameter, and the entropy density of the Universe at $T=m_{\mathrm{DM}}$, respectively, $Y=n / s$ is the DM yield [the ratio of the DM number density $(n)$ to the entropy density $(s)]$, $Y_{E Q}$ is the yield of the DM particle in thermal equilibrium, and $\left\langle\sigma v_{\text {rel }}\right\rangle$ is the thermal-averaged DM annihilation cross section times relative velocity $\left(v_{\text {rel }}\right)$. The formulas for the quantities in the Boltzmann equation are given as follows:

$$
\begin{aligned}
s(T) & =\frac{2 \pi^{2}}{45} g_{\star} T^{3}, \quad H(T)=\sqrt{\frac{\pi^{2}}{90} g_{\star}} \frac{T^{2}}{M_{P}}, \\
n_{E Q} & =s Y_{E Q}=\frac{g_{\mathrm{DM}}}{2 \pi^{2}} \frac{m_{\mathrm{DM}}^{3}}{x} K_{2}(x),
\end{aligned}
$$

where $M_{P}=2.43 \times 10^{18} \mathrm{GeV}$ is the reduced Planck mass, $g_{\mathrm{DM}}=1$ is the number of degrees of freedom for the
Higgs-portal DM, $g_{\star}$ is the effective number of total degrees of freedom for the particles in thermal equilibrium ( $g_{\star}=106.75$ for the SM particles), and $K_{2}$ is the modified Bessel function of the second kind. The thermal-averaged annihilation cross section is calculated by

$$
\begin{aligned}
\langle\sigma v\rangle= & \frac{1}{n_{E Q}} g_{\mathrm{DM}}^{2} \frac{m_{\mathrm{DM}}}{64 \pi^{4} x} \int_{4 m_{\mathrm{DM}}^{2}}^{\infty} d s 2\left(s-4 m_{\mathrm{DM}}^{2}\right) \\
& \times \sigma(s) \sqrt{s} K_{1}\left(\frac{x \sqrt{s}}{m_{\mathrm{DM}}}\right),
\end{aligned}
$$

where $\sigma(s)$ is the DM pair annihilation cross section corresponding to the processes in Fig. 3, and $K_{1}$ is the modified Bessel function of the first kind. By using the asymptotic value of the yield $Y(\infty)$, the DM relic density $\Omega_{\mathrm{DM}} h^{2}$ is expressed by

$$
\Omega_{\mathrm{DM}} h^{2}=\frac{m_{\mathrm{DM} s_{0}} Y(\infty)}{\rho_{c} / h^{2}},
$$

where $s_{0}=2890 \mathrm{~cm}^{-3}$ is the entropy density of the present Universe, and $\rho_{c} / h^{2}=1.05 \times 10^{-5} \mathrm{GeV} \mathrm{cm}^{-3}$ is the critical density.

The resultant DM relic density is controlled by two free parameters ( $m_{\mathrm{DM}}$ and $\left.\lambda_{H S S}\right)$, and their relation is determined so as to reproduce the observed DM relic density of $\Omega_{\mathrm{DM}} h^{2}=0.12$ [16]. In addition to the DM relic density, the parameter space of $m_{\mathrm{DM}}$ and $\lambda_{H S S}$ are constrained by the direct/indirect DM particle search results and the Higgsportal DM search results by the Large Hadron Collider (LHC) experiment. After all the constraints are taken into account, the allowed parameter region is identified. For the result, see, for example, Fig. 19 in Ref. [12]. It has been found that the Higgs-portal DM scenario is 
phenomenologically viable, but the allowed parameter region is very limited: $m_{\mathrm{DM}} \simeq M_{h} / 2$ with the SM Higgs boson mass $M_{h}=125 \mathrm{GeV}$ and $10^{-4} \lesssim\left|\lambda_{H S S}\right| \lesssim 10^{-3}$.

Now we introduce an SM-singlet chiral superfield $\Phi$ along with a $Z_{2}$ symmetry and assign odd parity to it while even parity to all the MSSM fields. Hence, the lightest component field in $\Phi$ is stable and the DM candidate. The SUSY Lagrangian $\mathcal{L}_{0}$ in Eq. (6) is then extended to be

$\mathcal{L}_{0} \rightarrow \mathcal{L}_{0}+\int d^{4} \theta \Phi^{\dagger} \Phi+\left\{\int d^{2} \theta \mu_{\Phi} \Phi^{2}+\right.$ H.c. $\}$,

where $\mu_{\Phi}$ is a mass parameter. Similar to $\mathcal{L}_{H}$ and $\mathcal{L}_{m}$, a new Lagrangian for $\Phi$ involving the Goldstino chiral superfield is given by

$$
\mathcal{L}_{\Phi}=-\frac{m_{\Phi}^{2}}{f^{2}} \int d^{4} \theta\left(X^{\dagger} X\right) \Phi^{\dagger} \Phi
$$

where $m_{\Phi}$ denotes a soft SUSY breaking mass. Finally, $\mathcal{L}_{A B}$ is extended to be

$$
\mathcal{L}_{A B} \rightarrow \mathcal{L}_{A B}+\left\{-\frac{B_{\Phi}}{2 f} \int d^{2} \theta X \Phi^{2}+\text { H.c. }\right\} .
$$

In the following, we assume that $B_{\Phi}$ is real and positive.
We now read off the scalar potential relevant to the Higgs-portal DM scenario by eliminating the auxiliary fields,

$$
V=V_{\mathrm{SUSY}}+V_{\mathrm{soft}},
$$

where

$$
\begin{aligned}
V_{\text {SUSY }}= & \mu_{H}^{2}\left(\left|H_{u}\right|^{2}+\left|H_{d}\right|^{2}\right)+\mu_{\Phi}^{2}|\Phi|^{2} \\
& +\frac{g_{Z}^{2}}{8}\left(\left|H_{u}\right|^{2}-\left|H_{d}\right|^{2}\right)^{2}+\frac{g_{2}^{2}}{2}\left|H_{u}^{\dagger} H_{d}\right|^{2}, \\
V_{\text {soft }} & =\frac{\left|f+\frac{m_{3}^{2}}{f} H_{u} H_{d}-\frac{B_{\Phi}}{2 f} \Phi^{2}\right|^{2}}{1-\frac{m_{1}^{2}}{f^{2}}\left|H_{d}\right|^{2}-\frac{m_{2}^{2}}{f^{2}}\left|H_{u}\right|^{2}-\frac{m_{\Phi}^{2}}{f^{2}}|\Phi|^{2}} .
\end{aligned}
$$

Although the complete form of the scalar potential includes all the sfermions in the MSSM, we have considered the potential terms involving only the MSSM Higgs doublets and the SM-singlet scalar $\Phi$. This is because the sfermions should be heavy to satisfy the current LHC constraints and their couplings with the Higgs-portal DM have little effects on the DM physics for $m_{\mathrm{DM}} \simeq M_{h} / 2$. For the physics of the Higgs-portal DM scenario, only the bilinear terms with respect to $\Phi$ are important. To extract them from the scalar potential, we expand $V_{\text {soft }}$ up to the order of $\mathcal{O}\left(1 / f^{2}\right)$ and then obtain

$$
\begin{aligned}
V \supset & {\left[\left(\mu_{\Phi}^{2}+m_{\Phi}^{2}\right)+\left\{\left(\frac{m_{3}^{2}}{f^{2}} H_{u} H_{d}+\text { H.c. }\right)+2 \frac{m_{1}^{2}}{f^{2}}\left|H_{d}\right|^{2}+2 \frac{m_{2}^{2}}{f^{2}}\left|H_{u}\right|^{2}\right\} m_{\Phi}^{2}\right]|\Phi|^{2} } \\
& -\left\{\left(1+\frac{m_{3}^{2}}{f^{2}} H_{u} H_{d}+\frac{m_{1}^{2}}{f^{2}}\left|H_{d}\right|^{2}+\frac{m_{2}^{2}}{f^{2}}\left|H_{u}\right|^{2}\right) \frac{B_{\Phi}}{2} \Phi^{2}+\text { H.c. }\right\} .
\end{aligned}
$$

Substituting

$$
\Phi=\frac{1}{\sqrt{2}}(\phi+i \eta)
$$

into Eq. (35), we can find the mass spectrum of the real scalars, $\phi$ and $\eta$, and their couplings with the Higgs bosons. First, we obtain the mass spectrum to be

$$
\begin{aligned}
m_{\phi / \eta}^{2}= & \mu_{\Phi}^{2}+m_{\Phi}^{2}+\left(\frac{m_{1}^{2}}{f} \cos ^{2} \beta+\frac{m_{2}^{2}}{f} \sin ^{2} \beta+\frac{m_{3}^{2}}{f} \sin \beta \cos \beta\right) \frac{m_{\Phi}^{2}}{f} v^{2} \\
& \mp\left\{1+\left(\frac{m_{1}^{2}}{f} \cos ^{2} \beta+\frac{m_{2}^{2}}{f} \sin ^{2} \beta+\frac{m_{3}^{2}}{f} \sin \beta \cos \beta\right) \frac{v^{2}}{f}\right\} B_{\Phi} \\
\simeq & \mu_{\Phi}^{2}+m_{\Phi}^{2} \mp B_{\Phi} .
\end{aligned}
$$

In the last expression, we have used $\left|m_{1,2,3}^{2}\right|, f \gg v^{2}$ and $m_{\Phi}^{2}<f$ from the theoretical consistency. We see that $m_{\phi}<m_{\eta}$, and thus, the real scalar $\phi$ is the DM candidate.
Since all the Higgs bosons except for the SM-like Higgs boson are heavy, the DM physics is mainly controlled by the coupling of $\phi$ with the SM-like Higgs boson. For a large $\tan \beta$ value, such as $\tan \beta=10$ as we have used in 
Figs. 1 and 2, the up-type Higgs doublet is approximately identified as the SM-like Higgs doublet. By employing this approximation $H_{u} \simeq H$, we can easily extract the coupling of $\phi$ with the SM-like Higgs doublet from Eq. (35) such that

$$
\mathcal{L}_{\mathrm{int}} \simeq-\frac{m_{2}^{2}}{f^{2}}\left(m_{\Phi}^{2}-\frac{B_{\Phi}}{2}\right)\left(H^{\dagger} H\right) \phi^{2}
$$

This is the formula to be compared with Eq. (22) with the identification of $S=\phi$. Therefore, in the decoupling limit of the heavy Higgs bosons and all the MSSM sparticles, we have obtained the Higgs-portal DM scenario as the low energy effective theory. In terms of our model parameters, the two parameters $m_{\mathrm{DM}}=m_{\phi}$ and $\lambda_{H S S}$, which control the Higgs-portal DM physics, are approximately expressed as

$$
\begin{aligned}
& m_{\mathrm{DM}}^{2} \simeq \mu_{\phi}^{2}+m_{\Phi}^{2}-B_{\Phi}, \\
& \lambda_{H S S} \simeq 4 \frac{m_{2}^{2}}{f^{2}}\left(m_{\Phi}^{2}-\frac{B_{\Phi}}{2}\right) .
\end{aligned}
$$

As in Fig. 1, we may fix $m_{2}^{2}=-(2005)^{2} \mathrm{GeV}^{2}$ and $\sqrt{f} \geq$ $3990 \mathrm{GeV}$ so as to yield $m_{h} \leq 125 \mathrm{GeV}$ at the tree level. Even after this choice, we still have three free parameters, $\mu_{\Phi}, m_{\Phi}$, and $B_{\Phi}$, and we can arrange them to satisfy the phenomenological constraints, $m_{\mathrm{DM}} \simeq M_{h} / 2$ and $10^{-4} \lesssim$ $\left|\lambda_{H S S}\right| \lesssim 10^{-3}$ for the Higgs-portal DM scenario. ${ }^{3}$ For example, we may set $\mu_{\Phi}^{2} \simeq m_{\Phi}^{2} \simeq B_{\Phi} / 2=\mathcal{O}\left(1 \mathrm{TeV}^{2}\right)$ but tune their differences so as to reproduce the allowed values of $m_{\mathrm{DM}}^{2} \ll 1 \mathrm{TeV}^{2}$ and $\left|\lambda_{H S S}\right| \ll 1$.

\section{CONCLUSION}

If SUSY is broken at a low energy, the NL-MSSM with the Goldstino chiral superfield is a very useful description for taking the hidden sector effect into account to the MSSM. The NL-MSSM may be particularly interesting if the SUSY breaking scale lies in the multi-TeV range. In this case, the SM-like Higgs boson mass $m_{h}=125 \mathrm{GeV}$ is achieved by the Higgs potential at the tree level after eliminating the $F$ component of the Goldstino superfield. However, such a low scale SUSY breaking predicts a milli-eV gravitino LSP, which is too light to be the main component of the DM in our Universe. Thus, a suitable DM candidate is missing in the NL-MSSM. To solve this problem, we have proposed a minimal extension of the NL-MSSM by introducing the SM-singlet chiral superfield $(\Phi)$ along with the $Z_{2}$ symmetry. The stability of the lightest component field in $\Phi$ is ensured by assigning

\footnotetext{
${ }^{3}$ For a parameter choice to predict $m_{h}<125 \mathrm{GeV}$ at the tree level, $M_{h}=125 \mathrm{GeV}$ should be reproduced by $M_{h}^{2}=m_{h}^{2}+$ $\Delta m_{h}^{2}$ with $\Delta m_{h}^{2}$ from quantum corrections through scalar top quarks, as usual in the MSSM.
}

odd parity to $\Phi$ while even parity for all the MSSM superfields. We have shown that in the decoupling limit of the sparticles and heavy Higgs bosons, our low energy effective theory is nothing but the Higgs-portal DM scenario with the lightest $Z_{2}$-odd real scalar being the DM candidate. With a suitable choice of the model parameters, we can reproduce the allowed parameter region of the Higgs-portal DM scenario.

Here, we give a comment on a general property of our model. Since the main point of this paper is to propose the minimal extension of the NL-MSSM to incorporate a suitable DM candidate, we have focused on a special parameter region, for which our model at low energies is reduced to the simplest Higgs-portal DM scenario (plus a extremely light gravitino), namely, the SM with the real scalar DM being odd under the $Z_{2}$ symmetry. In general, we have a wide variety of the parameter choices to realize a viable dark matter scenario. For example, we may take a very small value of $B_{\Phi}$ in Eq. (37) so that the mass splitting between $\phi$ and $\eta$ is negligibly small. In this case, we identify the complex scalar $\Phi$ with the DM particle. This is a complex scalar extension of the simplest Higgsportal DM scenario with only one real scalar. This extension has been studied in [10,17-22]. Since the MSSM includes two Higgs doublets, our Higgs-portal DM scenario is basically two Higgs doublet extension of the Higgs-portal DM scenario. The two Higgs doublet extension of the SM supplemented by the Higgs-portal DM has been studied in [23-38]. In the model, the heavy Higgs bosons can play an important role for the DM physics, for example, an enhancement of the DM pair annihilations through the heavy Higgs boson resonances. While the allowed parameter region of the simplest Higgs-portal DM scenario is very limited, a wide parameter space can be phenomenologically viable in the twoHiggs doublet extension. Although we focused on the interaction between the DM particle and the Higgs boson, the full Lagrangian includes interactions between the DM particles and sfermions, which are also derived by integrating out the $F$ component of the Goldstino superfield. If the DM particle is heavier than sfermions, the DM pair annihilation processes through the interaction between the DM particle and sfermions become important in evaluating the DM relic density. We leave such general analysis for future work.

Finally, let us consider a crucial difference of our model from standard neutralino dark matter scenario in the MSSM. Neutralino dark matter is an $R$-parity odd particle, while in our scenario the dark matter is an $R$-parity even particle. This fact leads to distinctive phenomenologies. For example, in collider phenomenology, a neutralino dark matter is produced through a cascade decay of heavier sparticles due to the $R$-parity conservation. On the other hand, Higgs-portal dark matter in our scenario is not produced by sparticle decays. A pair of Higgs-portal dark 
matters can be produced from the decays of Higgs bosons. Since gravitino is an $R$-parity odd particle, it is produced from a cascade decay of sparticles. In our scenario, the gravitino is almost massless, and missing energy distributions associated with gravitino production are very different from those associated with neutralino production.

\section{ACKNOWLEDGMENTS}

N. O. would like to thank the High Energy Theory Group in Yamagata University for the hospitality during his visit. This work is supported in part by the United States Department of Energy Grant No. DE-SC0012447 (N. O.).
[1] A. Brignole, F. Feruglio, and F. Zwirner, Aspects of spontaneously broken $\mathrm{N}=1$ global supersymmetry in the presence of gauge interactions, Nucl. Phys. B501, 332 (1997); H. Itoh, N. Okada, and T. Yamashita, Low scale gravity mediation with warped extra dimension and collider phenomenology on the hidden sector, Phys. Rev. D 74, 055005 (2006); M. Dine, N. Seiberg, and S. Thomas, Higgs physics as a window beyond the MSSM (BMSSM), Phys. Rev. D 76, 095004 (2007); I. Antoniadis, E. Dudas, D. M. Ghilencea, and P. Tziveloglou, MSSM with dimension-five operators (MSSM(5)), Nucl. Phys. B808, 155 (2009); MSSM Higgs with dimension-six operators, Nucl. Phys. B831, 133 (2010); H. Murayama, Y. Nomura, S. Shirai, and K. Tobioka, Compact supersymmetry, Phys. Rev. D 86, 115014 (2012).

[2] M. Rocek, Linearizing the Volkov-Akulov Model, Phys. Rev. Lett. 41, 451 (1978).

[3] U. Lindstrom and M. Rocek, Constrained local superfields, Phys. Rev. D 19, 2300 (1979).

[4] Z. Komargodski and N. Seiberg, From linear SUSY to constrained superfields, J. High Energy Phys. 09 (2009) 066.

[5] I. Antoniadis, E. Dudas, D. M. Ghilencea, and P. Tziveloglou, Non-linear MSSM, Nucl. Phys. B841, 157 (2010).

[6] I. Antoniadis, E. Dudas, D. M. Ghilencea, and P. Tziveloglou, Nonlinear supersymmetry and Goldstino couplings to the MSSM, Theor. Math. Phys. 170, 26 (2012).

[7] I. Antoniadis, E. M. Babalic, and D. M. Ghilencea, Naturalness in low-scale SUSY models and "non-linear" MSSM, Eur. Phys. J. C 74, 3050 (2014).

[8] E. Dudas, C. Petersson, and P. Tziveloglou, Low scale supersymmetry breaking and its LHC signatures, Nucl. Phys. B870, 353 (2013).

[9] J. L. Feng, M. Kamionkowski, and S. K. Lee, Light gravitinos at colliders and implications for cosmology, Phys. Rev. D 82, 015012 (2010).

[10] J. McDonald, Gauge singlet scalars as cold dark matter, Phys. Rev. D 50, 3637 (1994).

[11] C. P. Burgess, M. Pospelov, and T. ter Veldhuis, The minimal model of nonbaryonic dark matter: A singlet scalar, Nucl. Phys. B619, 709 (2001).

[12] G. Arcadi, A. Djouadi, and M. Raidal, Dark Matter through the Higgs portal, Phys. Rep. 842, 1 (2020).

[13] D. V. Volkov and V. P. Akulov, Is the neutrino a goldstone particle? Phys. Lett. 46B, 109 (1973).

[14] S. P. Martin, A supersymmetry primer, Adv. Ser. Dir. High Energy Phys. 21, 1 (2010).
[15] P. Gondolo and G. Gelmini, Cosmic abundances of stable particles: Improved analysis, Nucl. Phys. B360, 145 (1991).

[16] N. Aghanim et al. (Planck Collaboration), Planck 2018 results. VI. Cosmological parameters, Astron. Astrophys. 641, A6 (2020).

[17] V. Barger, M. McCaskey, and G. Shaughnessy, Complex scalar dark matter vis-à-vis CoGeNT, DAMA/LIBRA and XENON100, Phys. Rev. D 82, 035019 (2010).

[18] M. Gonderinger, H. Lim, and M. J. Ramsey-Musolf, Complex scalar singlet dark matter: Vacuum stability and phenomenology, Phys. Rev. D 86, 043511 (2012).

[19] C. W. Chiang, T. Nomura, and J. Tandean, Dark matter and Higgs boson in a model with discrete gauge symmetry, Phys. Rev. D 87, 073004 (2013).

[20] R. Coimbra, M. O. P. Sampaio, and R. Santos, ScannerS: Constraining the phase diagram of a complex scalar singlet at the LHC, Eur. Phys. J. C 73, 2428 (2013).

[21] R. Costa, M. Mühlleitner, M. O. P. Sampaio, and R. Santos, Singlet extensions of the Standard Model at LHC Run 2: Benchmarks and comparison with the NMSSM, J. High Energy Phys. 06 (2016) 034.

[22] H. Wu and S. Zheng, Scalar dark matter: Real vs complex, J. High Energy Phys. 03 (2017) 142.

[23] C. Bird, R. V. Kowalewski, and M. Pospelov, Dark matter pair-production in $\mathrm{b} \longrightarrow \mathrm{s}$ transitions, Mod. Phys. Lett. A 21, 457 (2006).

[24] X. G. He, T. Li, X. Q. Li, and H. C. Tsai, Scalar dark matter effects in Higgs and top quark decays, Mod. Phys. Lett. A 22, 2121 (2007).

[25] X. G. He, T. Li, X. Q. Li, J. Tandean, and H. C. Tsai, Constraints on scalar dark matter from direct experimental searches, Phys. Rev. D 79, 023521 (2009).

[26] B. Grzadkowski and P. Osland, Tempered two-Higgsdoublet model, Phys. Rev. D 82, 125026 (2010).

[27] M. Aoki, S. Kanemura, and O. Seto, Multi-Higgs portal dark matter under the CDMS II results, Phys. Lett. B 685 , 313 (2010).

[28] T. Li and Q. Shafi, Scalar dark matter search at the LHC through FCNC top decay, Phys. Rev. D 83, 095017 (2011).

[29] Y. Cai, X. G. He, and B. Ren, Low mass dark matter and invisible Higgs width in darkon models, Phys. Rev. D 83, 083524 (2011).

[30] X. G. He, B. Ren, and J. Tandean, Hints of Standard Model Higgs Boson at the LHC and light dark matter searches, Phys. Rev. D 85, 093019 (2012). 
[31] Y. Bai, V. Barger, L. L. Everett, and G. Shaughnessy, TwoHiggs-doublet-portal dark-matter model: LHC data and Fermi-LAT 135 GeV line, Phys. Rev. D 88, 015008 (2013).

[32] X. G. He and J. Tandean, Low-mass dark-matter hint from CDMS II, Higgs boson at the LHC, and darkon models, Phys. Rev. D 88, 013020 (2013).

[33] A. Greljo, J. Julio, J. F. Kamenik, C. Smith, and J. Zupan, Constraining Higgs mediated dark matter interactions, J. High Energy Phys. 11 (2013) 190.

[34] L. Wang and X. F. Han, A simplified 2HDM with a scalar dark matter and the galactic center gamma-ray excess, Phys. Lett. B 739, 416 (2014).

[35] A. Drozd, B. Grzadkowski, J. F. Gunion, and Y. Jiang, Extending two-Higgs-doublet models by a singlet scalar
field-The case for dark matter, J. High Energy Phys. 11 (2014) 105.

[36] N. Okada and O. Seto, Galactic center gamma-ray excess from two-Higgs-doublet-portal dark matter, Phys. Rev. D 90, 083523 (2014).

[37] R. Campbell, S. Godfrey, H. E. Logan, A. D. Peterson, and A. Poulin, Implications of the observation of dark matter self-interactions for singlet scalar dark matter, Phys. Rev. D 92, 055031 (2015); Erratum, Phys. Rev. D 101, 039905 (2020).

[38] A. Drozd, B. Grzadkowski, J. F. Gunion, and Y. Jiang, Isospin-violating dark-matter-nucleon scattering via twoHiggs-doublet-model portals, J. Cosmol. Astropart. Phys. 10 (2016) 040. 